\title{
Further Results on Lyapunov Functions for Slowly Time-Varying Systems
}

\author{
Frédéric Mazenc* Michael Malisoff ${ }^{\dagger}$
}

August 3, 2018

\begin{abstract}
We provide general methods for explicitly constructing strict Lyapunov functions for fully nonlinear slowly time-varying systems. Our results apply to cases where the given dynamics and corresponding frozen dynamics are not necessarily exponentially stable. This complements our previous Lyapunov function constructions for rapidly time-varying dynamics. We also explicitly construct input-to-state stable Lyapunov functions for slowly time-varying control systems. We illustrate our findings by constructing explicit Lyapunov functions for a pendulum model, an example from identification theory, and a perturbed friction model.
\end{abstract}

Key Words: Lyapunov function constructions, slowly time-varying systems, stability analysis, input-to-state stability

\section{Introduction}

This paper is devoted to the study of fully nonlinear slowly time-varying systems of the form

$$
\dot{x}=f(x, t, t / \alpha)
$$

for large values of the constant $\alpha>0$ (but see Section 7 below for the extension to systems with controls). See Section 2 for our standing assumptions on (11). Such systems arise in a large variety of important engineering applications such as the control of friction and pendulums [K, PA2, S1]. It is therefore of great interest in control engineering to develop methods for determining whether slowly time-varying systems are uniformly globally asymptotically stable (UGAS). When (11) is UGAS, it is also highly desirable to have general methods for constructing explicit closed form Lyapunov functions for (1). See for example [A, AS, ASW, M, MM3, MMD] for discussions on the essentialness of Lyapunov functions for feedback design and robustness analysis. See also [MMD, PA1] for the dual problem of stabilizing rapidly time-varying systems, and see Remark 8 below for the relationship between our methods for constructing Lyapunov functions for rapidly and slowly time-varying systems.

One popular approach to studying (1) is to first establish exponential stability of the corresponding "frozen dynamics"

$$
\dot{x}=f(x, t, \tau)
$$

${ }^{*}$ Mazenc is with Projet MERE INRIA-INRA, UMR Analyse des Systèmes et Biométrie INRA, 2, pl. Viala, 34060 Montpellier, France (email: Frederic.Mazenc@ensam.inra.fr).

${ }^{\dagger}$ Malisoff is with the Department of Mathematics, Louisiana State University, Baton Rouge, LA 70803-4918 USA (email: malisoff@lsu.edu). 
for all relevant values of the parameter $\tau$ including cases where the exponent in the exponential decay estimate can be negative or positive for some values of $\tau$ but is positive on average [K, $\mathrm{PA}$, , S1]. The stability of the frozen dynamics is then used to establish stability of (1). However, these earlier results do not lead to explicit Lyapunov functions for (11) that would be needed for robustness analysis. The main goals of our work are (i) to show that explicit Lyapunov functions for (1) can be explicitly constructed in terms of a suitable class of oftentimes readily available Lyapunov functions for (2) when the constant $\alpha>0$ is large enough, and (ii) to show how to relax the exponential like stability assumptions on (2) and also allow cases where $\tau$ is a vector, thereby broadening the class of dynamics to which the frozen dynamics method can be applied.

The rest of this paper is organized as follows. In Section 2 we provide the relevant definitions and standing assumptions on (11). We state and prove our main result in Sections 34. In Section 5 , we extend our main result to cases where the Lyapunov functions for the frozen systems satisfy less restrictive properties than those in Sections 3, 4. In Section 6, we illustrate the wide applicability of our results using four examples. In the first two examples, the family of Lyapunov like functions for (2) are independent of $\tau$, so the strict Lyapunov functions we construct for (1) are valid for all $\alpha>0$ i.e. (1) is UGAS for all $\alpha>0$. Our next two examples involve a mass spring model with slowly time-varying coefficients from [DDNZ] and an identification model similar to those studied in [PA1, and illustrate the more general situation where (1) is not necessarily UGAS for all values of $\alpha>0$. In each case, the dynamics have slowly time-varying coefficients and so are beyond the scope of the previously known Lyapunov construction methods. In Section 7, we show how to extend our results to systems with controls using input-to-state stability. We close in Section 8 with some suggestions for further research.

\section{Definitions, Assumptions, and Lemmas}

We let $\mathcal{K}_{\infty}$ denote the set of all continuous functions $\rho:[0, \infty) \rightarrow[0, \infty)$ for which (i) $\rho(0)=0$ and (ii) $\rho$ is strictly increasing and unbounded. We let $\mathcal{K} \mathcal{L}$ denote the class of all continuous functions $\beta:[0, \infty) \times[0, \infty) \rightarrow[0, \infty)$ for which

(I) $\beta(\cdot, t) \in \mathcal{K}_{\infty}$ for each $t \geq 0$,

(II) $\beta(s, \cdot)$ is non-increasing for each $s \geq 0$, and

(III) $\beta(s, t) \rightarrow 0$ as $t \rightarrow+\infty$ for each $s \geq 0$.

When we say that a function $\rho$ is smooth (a.k.a. $C^{1}$ ), we mean it is continuously differentiable, written $\rho \in C^{1}$. (For functions $\rho$ defined on $[0, \infty)$, we interpret $\rho^{\prime}(0)$ as a one-sided derivative, and continuity of $\rho^{\prime}$ at 0 as one-sided continuity.) We let $|\cdot|$ denote the Euclidean norm. A continuous function $\rho:[0, \infty) \rightarrow[0, \infty)$ is called positive definite provided it is zero only at zero. When $r \mapsto p(r) \in \mathbb{R}^{d}$ is a function with differentiable components, we use $p^{\prime}(r)$ to denote the vector $\left(p_{1}^{\prime}(r), \ldots, p_{d}^{\prime}(r)\right)$.

The following definitions and lemma apply to general nonlinear systems

$$
\dot{x}=h(x, t)
$$

evolving on the state space $\mathbb{R}^{n}$ where $h$ is locally Lipschitz (but see Section 7 for the extension to control systems). Later we specialize to systems with multiple time scales and frozen parameters, e.g. $h(x, t)=f(x, t, p(t / \alpha))$ or $h(x, t)=f(x, t, \tau)$ for given constant parameters $\alpha$ and $\tau$ and 
suitable functions $p$. We always assume (3) is forward complete meaning for each $x_{o} \in \mathbb{R}^{n}$ and $t_{o} \in \mathbb{R}_{\geq 0}:=[0, \infty)$ there exists a unique trajectory

$$
\left[t_{0}, \infty\right) \ni t \mapsto \phi\left(t ; t_{o}, x_{o}\right)
$$

for (3) that satisfies $x\left(t_{o}\right)=x_{o}$. We assume all of our uncontrolled dynamics (3) are uniformly state bounded meaning there exists $\alpha_{h} \in \mathcal{K}_{\infty}$ such that $|h(x, t)| \leq \alpha_{h}(|x|)$ everywhere.

Definition 1 We say that (3) is uniformly globally asymptotically stable (UGAS) provided there exists $\beta \in \mathcal{K} \mathcal{L}$ such that

$$
\left|\phi\left(t ; t_{o}, x_{o}\right)\right| \leq \beta\left(\left|x_{o}\right|, t-t_{o}\right)
$$

for all $x_{o} \in \mathbb{R}^{n}, t_{o} \in[0, \infty)$, and $t \geq t_{o}$.

Definition 2 A smooth function $W: \mathbb{R}^{n} \times[0, \infty) \rightarrow[0, \infty)$ is called a Lyapunov function for (3) provided there are functions $\alpha_{1}, \alpha_{2} \in \mathcal{K}_{\infty}$ and a positive definite function $\alpha_{3}$ such that

(L1) $\quad \alpha_{1}(|x|) \leq W(x, t) \leq \alpha_{2}(|x|)$ and

(L2) $W_{t}(x, t)+W_{x}(x, t) h(x, t) \leq-\alpha_{3}(|x|)$

hold for all $t \geq 0$ and $x \in \mathbb{R}^{n}$.

The subscripts on $W$ denote partial gradients. In what follows, we often omit the arguments $x, t$, etc. in our functions when they are clear from the context; and all (in)equalities should be interpreted to hold wherever they make sense. A smooth function $W: \mathbb{R}^{n} \times[0, \infty) \rightarrow[0, \infty)$ that admits $\alpha_{1}, \alpha_{2} \in \mathcal{K}_{\infty}$ such that (L1) holds everywhere is called uniformly proper and positive definite. The following lemma is standard [ELW, $\mathrm{K}]$ :

Lemma 3 If (3) admits a Lyapunov function, then it is UGAS.

A simple application of Fubini's Theorem yields the formula

$$
\int_{t-c}^{t} \int_{s}^{t} \Theta(l) d l d s=\int_{t-c}^{t}(r-t+c) \Theta(r) d r
$$

and therefore also the following [MM3]:

Lemma 4 Let $\Theta: \mathbb{R} \rightarrow \mathbb{R}$ be continuous and bounded in norm by some constant $\bar{M}>0$, and $c>0$ be given. Then

$$
\left|\int_{t-c}^{t} \int_{s}^{t} \Theta(l) d l d s\right| \leq \frac{c^{2} \bar{M}}{2} \quad \text { and }
$$

(B) $\frac{d}{d t} \int_{t-c}^{t} \int_{s}^{t} \Theta(l) d l d s=c \Theta(t)-\int_{t-c}^{t} \Theta(r) d r$

hold for all $t \in \mathbb{R}$. 


\section{Statement of Main Result and Remarks}

For simplicity, we assume our system (1) has the form

$$
\dot{x}=f(x, t, p(t / \alpha))
$$

where $p: \mathbb{R} \rightarrow \mathbb{R}^{d}$ (for some integer $d$ ) is bounded and its components $p_{1}, \ldots, p_{d}$ have bounded first derivatives. We set

$$
\bar{p}:=\sup \left\{\left|p^{\prime}(r)\right|: r \in \mathbb{R}\right\} \quad \text { and } \quad \mathcal{R}(p):=\{p(t): t \in \mathbb{R}\} .
$$

Our next assumption is a variant of those of [PA2, Theorem 2] (but see Section 5 for results under weaker assumptions).

Assumption 5 There exist $\alpha_{1}, \alpha_{2} \in \mathcal{K}_{\infty}$, positive constants $c_{a}, c_{b}$, and $T$, a continuous function $q: \mathbb{R}^{d} \rightarrow \mathbb{R}$, and a $C^{1}$ function $V: \mathbb{R}^{n} \times[0, \infty) \times \mathbb{R}^{d} \rightarrow[0, \infty)$ such that

$A_{1} \quad \alpha_{1}(|x|) \leq V(x, t, \tau) \leq \alpha_{2}(|x|)$,

$A_{2} V_{t}(x, t, \tau)+V_{x}(x, t, \tau) f(x, t, \tau) \leq-q(\tau) V(x, t, \tau)$,

$A_{3}\left|V_{\tau}(x, t, \tau)\right| \leq c_{a} V(x, t, \tau)$, and

$A_{4} \int_{t-T}^{t} q(p(s)) d s \geq c_{b}$

hold for all $x \in \mathbb{R}^{n}, t \geq 0$, and $\tau \in \mathcal{R}(p)$.

Note that $A_{2}$ is weaker than the standard exponential stability property of the frozen dynamics since we do not require $\alpha_{1}$ or $\alpha_{2}$ to be quadratic functions and moreover $q(\tau)$ can take non-positive values for some choices of the vector parameter $\tau$. However, $A_{4}$ requires that $q$ be positive on average along the vector $p(s)$.

Theorem 6 If (4) satisfies Assumption 5, then for each constant $\alpha>2 T c_{a} \bar{p} / c_{b}$, the dynamics (4) are $U G A S$ and

$$
V_{\alpha}^{\sharp}(t, x):=e^{\frac{\alpha}{T} \int_{\frac{t}{\alpha}-T}^{\frac{t}{\alpha}} \int_{s}^{\frac{t}{\alpha}} q(p(l)) d l d s} V(x, t, p(t / \alpha))
$$

is a Lyapunov function for (4).

Remark 7 Compared to the known results [K, PA2, [S1], the novelty of Theorem [6 is that (a) we allow fully nonlinear systems including cases where the function $q$ can take both positive and negative values (which corresponds to the allowance in [S1] of eigenvalues that wander into the right half plane while remaining in the strict left half plane on average) and (b) we provide an explicit Lyapunov function (5) for the original slowly time-varying dynamics. In general, the conclusion of Theorem [6 may or may not hold for small values of $\alpha$. We illustrate this in Section [6 below.

Remark 8 A completely different approach to slowly time-varying systems $\dot{x}=f(x, t, t / \alpha)$ (for large constants $\alpha>0$ ) is to transform the system into a rapidly time-varying system and to then try to construct a Lyapunov function for the resulting rapidly time-varying system directly. The 
transformation is done by simply setting $s=t / \alpha$ which gives rise to the new rapidly time-varying system

$$
\dot{x}(s)=g(x(s), s, \alpha s):=\alpha f(x(s), s \alpha, s)
$$

in terms of the new rescaled time variable s. However, it might be difficult to apply the Lyapunov function construction methods of [MMD] or other known methods to build an explicit Lyapunov function for (6). This is because these earlier results are for fast time-varying dynamics having a different form from (6) and moreover they require Lyapunov functions for so-called limiting dynamics; cf. [PA1, Property 2], and see [MMD, Section 3.1] for a generalization in the same vein. This motivates our direct construction of Lyapunov functions for slowly time-varying dynamics, which may be viewed as a complementary approach to the time rescaling method since we do not require limiting dynamics.

\section{Proof of Theorem 6}

By $A_{2}-A_{3}$ and our choice of $\bar{p}$, the time derivative of

$$
\hat{V}(x, t):=V(x, t, p(t / \alpha))
$$

along the trajectories of (44) satisfies:

$$
\begin{aligned}
\dot{\hat{V}} & =V_{t}(x, t, p(t / \alpha))+V_{x}(x, t, p(t / \alpha)) f(x, t, p(t / \alpha))+V_{\tau}(x, t, p(t / \alpha)) \frac{p^{\prime}(t / \alpha)}{\alpha} \\
& \leq-q(p(t / \alpha)) \hat{V}(t, x)+V_{\tau}(x, t, p(t / \alpha)) \frac{p^{\prime}(t / \alpha)}{\alpha} \\
& \leq\left[-q(p(t / \alpha))+\frac{c_{a} \bar{p}}{\alpha}\right] \hat{V}(x, t) .
\end{aligned}
$$

To simplify the notation, let us define

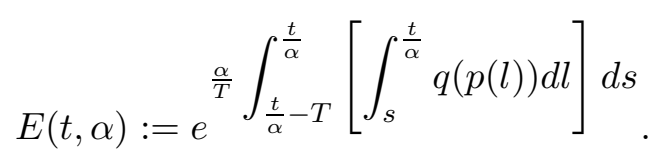

Since $p$ is bounded and $q$ is continuous,

$$
\Theta(t):=q(p(t))
$$

is bounded in norm by some constant $\bar{M}>0$. Therefore, along the trajectories of (44), Lemma 4 (B) with the choices (9) and $c=T$ gives

$$
\dot{V}_{\alpha}^{\sharp}=E(t, \alpha)\left[\dot{\hat{V}}+\left\{q(p(t / \alpha))-\frac{1}{T} \int_{\frac{t}{\alpha}-T}^{\frac{t}{\alpha}} q(p(l)) d l\right\} \hat{V}\right] .
$$

Substituting the formula for $\dot{\hat{V}}$, it follows from $A_{4}$ that

$$
\begin{aligned}
\dot{V}_{\alpha}^{\sharp} & \leq E(t, \alpha)\left[\frac{c_{a} \bar{p}}{\alpha}-\frac{1}{T} \int_{\frac{t}{\alpha}-T}^{\frac{t}{\alpha}} q(p(l)) d l\right] \hat{V} \\
& \leq E(t, \alpha)\left[\frac{c_{a} \bar{p}}{\alpha}-\frac{c_{b}}{T}\right] \hat{V}(x, t) .
\end{aligned}
$$


Applying Lemma 4 (A) with the choices (9) and $c=T$ gives

$$
e^{\alpha T \bar{M} / 2} \geq E(t, \alpha) \geq e^{-\alpha T \bar{M} / 2}
$$

everywhere. Hence, for $\alpha>2 T c_{a} \bar{p} / c_{b}$, (10) gives

$$
\dot{V}_{\alpha}^{\sharp}(x, t) \leq-\frac{c_{b}}{2 T} e^{-\alpha T \bar{M} / 2} \hat{V}(x, t) \leq-\alpha_{3}(|x|),
$$

where $\alpha_{3}(s)=\frac{c_{b}}{2 T} e^{-\alpha T \bar{M} / 2} \alpha_{1}(s)$ is positive definite; and

$$
\hat{\alpha}_{1}(|x|) \leq V_{\alpha}^{\sharp}(x, t) \leq \hat{\alpha}_{2}(|x|)
$$

everywhere, where

$$
\hat{\alpha}_{1}(s):=e^{-\alpha T \bar{M} / 2} \alpha_{1}(s), \quad \hat{\alpha}_{2}(s):=e^{\alpha T \bar{M} / 2} \alpha_{2}(s)
$$

are of class $\mathcal{K}_{\infty}$. Since (11)-(12) imply that $V_{\alpha}^{\sharp}$ is a Lyapunov function for (44), Theorem 6 follows from Lemma 3 .

\section{More General Families of Lyapunov Functions}

We next show how to relax requirements $A_{2}-A_{3}$ from Theorem 6 . We continue to use the notation we introduced in Section 3. We assume the following in the rest of this section:

Assumption 9 There exist $\tilde{\alpha}_{1}, \tilde{\alpha}_{2} \in \mathcal{K}_{\infty}$, a positive definite $C^{1}$ function $\mu$, positive constants $T$, $\tilde{c}_{a}$, and $\tilde{c}_{b}$, a continuous function $\tilde{q}: \mathbb{R}^{d} \rightarrow \mathbb{R}$, and a $C^{1}$ function $\tilde{V}: \mathbb{R}^{n} \times[0, \infty) \times \mathbb{R}^{d} \rightarrow[0, \infty)$ such that

$$
\lim _{r \rightarrow+\infty} \int_{1}^{r} \frac{1}{\mu(l)} d l=+\infty
$$

and

$$
\begin{aligned}
& \tilde{A}_{1} \quad \tilde{\alpha}_{1}(|x|) \leq \tilde{V}(x, t, \tau) \leq \tilde{\alpha}_{2}(|x|), \\
& \tilde{A}_{2} \quad \tilde{V}_{t}(x, t, \tau)+\tilde{V}_{x}(x, t, \tau) f(x, t, \tau) \leq-\tilde{q}(\tau) \mu(\tilde{V}(x, t, \tau)), \\
& \tilde{A}_{3} \quad\left|\tilde{V}_{\tau}(x, t, \tau)\right| \leq \tilde{c}_{a} \mu(\tilde{V}(x, t, \tau)), \text { and } \\
& \tilde{A}_{4} \quad \int_{t-T}^{t} \tilde{q}(p(s)) d s \geq \tilde{c}_{b}
\end{aligned}
$$

hold for all $x \in \mathbb{R}^{n}, t \geq 0$, and $\tau \in \mathcal{R}(p)$.

Notice that Assumption 5 is the special case of Assumption 9 in which $\mu(l) \equiv l$. We prove the following:

Theorem 10 If (4) satisfies Assumption 9, then there exists $k \in \mathcal{K}_{\infty}$ for which the requirements of Assumption 5 are satisfied with $V:=k(\tilde{V})$. Therefore, for each sufficiently large value of the constant $\alpha>0$, the dynamics (4) is UGAS and admits a Lyapunov function of the form (5).

It suffices to prove the first statement of Theorem 10 since the second statement is immediate from Theorem 6. To this end, we use the following important observation: 
Lemma 11 If $\mu \in C^{1}$ is positive definite, then

$$
\lim _{r \rightarrow 0^{+}} \int_{1}^{r} \frac{1}{\mu(l)} d l=-\infty
$$

Proof: Since $\mu \in C^{1}$ and $\mu(0)=0$, we can find $c_{3}>0$ such that $\mu(r) \leq c_{3} r$ for all $r \in[0,1]$. Hence, for each $r \in(0,1]$, we get

$$
\int_{r}^{1} \frac{1}{\mu(l)} d l \geq \int_{r}^{1} \frac{1}{c_{3} l} d l=-\frac{1}{c_{3}} \ln (r)
$$

It follows that, for all $r \in(0,1]$,

$$
\int_{1}^{r} \frac{1}{\mu(l)} d l \leq \frac{1}{c_{3}} \ln (r)
$$

Since $\lim _{r \rightarrow 0^{+}} \ln (r)=-\infty$, the lemma follows.

Given a constant $\xi>0$ which we specify later, it follows from (13) and (14) that

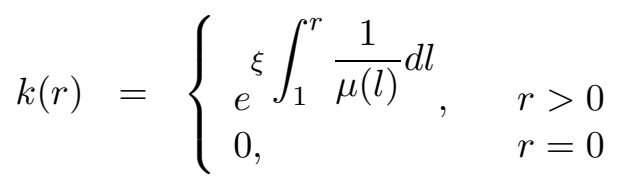

is continuous and unbounded. In particular, $k(r) \rightarrow 0$ as $r \rightarrow 0^{+}$. Set $B=\sup \left\{\mu^{\prime}(s): 0 \leq s \leq 1\right\}$, which is positive since $\mu$ is positive definite.

Lemma 12 The function (17) is $C^{1}$ when $\xi=2 B$.

Proof: It suffices to prove that

$$
k^{\prime}(r) \rightarrow 0 \text { as } 0<r \rightarrow 0^{+}
$$

since $k(0)=0$, because then $k(r) / r \rightarrow 0=k^{\prime}(0)$ as $r \rightarrow 0^{+}$. To this end, first note that

$$
k^{\prime}(r)=\frac{\xi}{\mu(r)} e^{-\xi \int_{r}^{1} \frac{1}{\mu(l)} d l}, \forall r>0
$$

and that for all $r \in(0,1]$, we have

$$
\frac{1}{B}[\ln (\mu(1))-\ln (\mu(r))]=\int_{r}^{1} \frac{\mu^{\prime}(l)}{B \mu(l)} d l \leq \int_{r}^{1} \frac{1}{\mu(l)} d l
$$

by our choice of $B$. Since $\mu$ is positive definite and $\xi$ is positive, this implies

$$
\frac{\xi}{\mu(r)} e^{\frac{-\xi}{B}[\ln (\mu(1))-\ln (\mu(r))]} \geq \frac{\xi}{\mu(r)} e^{-\xi \int_{r}^{1} \frac{1}{\mu(l)} d l}=k^{\prime}(r)
$$

for all $r \in(0,1]$, i.e.,

$$
\xi(\mu(1))^{\frac{-\xi}{B}} \mu(r)^{\frac{\xi}{B}-1} \geq k^{\prime}(r) \geq 0 \quad \forall r \in(0,1]
$$

so (18) follows from our choice of $\xi$. 
The fact that $k \in \mathcal{K}_{\infty} \cap C^{1}$ is now immediate from Assumption 9 and Lemmas 11 and [12, Let us now verify that $k$ satisfies the requirements of Theorem 10. From the definition of $k$, we deduce that

$$
k^{\prime}(\tilde{V}) \mu(\tilde{V})=\frac{2 B}{\mu(\tilde{V})} e^{-2 B} \int_{\tilde{V}}^{1} \frac{1}{\mu(l)} d l \quad \mu(\tilde{V})=2 B k(\tilde{V})
$$

when $\tilde{V} \neq 0$. Therefore, by assumption $\tilde{A}_{2}$, the time derivative of $V=k(\tilde{V})$ along the trajectories of (4) satisfies

$$
\begin{aligned}
V_{t}(x, t, \tau)+V_{x}(x, t, \tau) f(x, t, \tau) & =k^{\prime}(\tilde{V}(x, t, \tau))\left[\tilde{V}_{t}(x, t, \tau)+\tilde{V}_{x}(x, t, \tau) f(x, t, \tau)\right] \\
& \leq-k^{\prime}(\tilde{V}(x, t, \tau)) \tilde{q}(\tau) \mu(\tilde{V}(x, t, \tau)) \\
& =-2 B \tilde{q}(\tau) V(x, t, \tau)
\end{aligned}
$$

everywhere, and condition $\tilde{A}_{3}$ from Assumption 9 implies

$$
\left|V_{\tau}\right|=k^{\prime}(\tilde{V})\left|\tilde{V}_{\tau}\right| \leq \tilde{c}_{a} k^{\prime}(\tilde{V}) \mu(\tilde{V})=2 B \tilde{c}_{a} V
$$

everywhere. Therefore Assumption 5 holds using

$$
\alpha_{i}(s):=k \circ \alpha_{i}(s) \text { for } i=1,2, \quad V:=k(\tilde{V}), \quad q(\tau):=2 B \tilde{q}(\tau), \quad c_{a}:=2 B \tilde{c}_{a}, \quad c_{b}:=2 B \tilde{c}_{b} .
$$

The result now follows from Theorem 6 .

\section{$6 \quad$ Examples}

We illustrate our constructions using four examples. In the first two examples, the functions $V$ from Assumption 5 do not depend on the frozen parameter $\tau$, so we can conclude that (4) is UGAS for all values of the constant $\alpha>0$. We then turn to a slowly time-varying friction dynamics and an example from identification where $V$ depends on $\tau$, and where we can consequently only conclude the UGAS property of (4) when $\alpha>0$ is sufficiently large. Set $\dot{V}(x, t, \tau):=V_{t}(x, t, \tau)+$ $V_{x}(x, t, \tau) f(x, t, \tau)$ everywhere.

\subsection{Stability for all $\alpha>0$ : a scalar example}

Consider the one-dimensional system

$$
\dot{x}=\frac{x}{\sqrt{1+x^{2}}}\left[1-90 \cos ^{2}\left(\frac{t}{\alpha}\right)\right]
$$

and the uniformly proper and positive definite function

$$
V(x, t, \tau) \equiv \bar{V}(x):=e^{\sqrt{1+x^{2}}}-e .
$$

Let us verify Assumption 5 for this choice of $V$ and the frozen dynamics

$$
\dot{x}=f(x, t, \tau):=\frac{x}{\sqrt{1+x^{2}}}[1-90 \tau], \quad 0 \leq \tau \leq 1 .
$$


This gives

$$
\begin{aligned}
\dot{V}(x, t, \tau) & =e^{\sqrt{1+x^{2}}} \frac{x^{2}}{1+x^{2}}[1-90 \tau] \\
& =e^{\sqrt{1+x^{2}}} \frac{x^{2}}{1+x^{2}}-90 \tau e^{\sqrt{1+x^{2}}} \frac{x^{2}}{1+x^{2}} .
\end{aligned}
$$

Simple calculus calculations everywhere give

$$
\frac{2 e^{\sqrt{2}}}{e-1} \bar{V}(x) \geq \frac{x^{2}}{1+x^{2}} e^{\sqrt{1+x^{2}}} \geq \frac{1}{2} \bar{V}(x)
$$

so (25) everywhere gives

$$
\dot{V}(x, t, \tau) \leq\left[\frac{2 e^{\sqrt{2}}}{e-1}-45 \tau\right] \bar{V}(x) .
$$

Moreover, for each $t \geq 0$, we get

$$
\int_{t-\pi}^{t}\left[45 \cos ^{2}(s)-\frac{2 e^{\sqrt{2}}}{e-1}\right] d s=\pi\left(\frac{45}{2}-\frac{2 e^{\sqrt{2}}}{e-1}\right)>0
$$

which shows that Assumption 5 is satisfied. We conclude from Theorem 6 that for large enough constants $\alpha>0$, (22) is UGAS and has the Lyapunov function

$$
e^{\frac{\alpha}{\pi} \int_{\frac{t}{\alpha}-\pi}^{\frac{t}{\alpha}}\left[\int_{s}^{\frac{t}{\alpha}}\left[45 \cos ^{2}(l)-\frac{2 e^{\sqrt{2}}}{e-1}\right] d l\right] d s} \bar{V}(x)=e^{45 \frac{\alpha}{4}\left[\sin \left(\frac{2 t}{\alpha}\right)+\pi-\frac{4 \pi e^{\sqrt{2}}}{45(e-1)}\right]}\left[e^{\sqrt{1+x^{2}}}-e\right]
$$

where $\bar{V}$ is in (23). In fact, since in this case $V$ does not depend on $\tau$, it follows from our proof of Theorem [6 that for any constant $\alpha>0$, the system (22) is UGAS and admits the Lyapunov function (28).

Remark 13 The dynamics (22) illustrates the fact that our approach applies to systems which are not globally exponentially stable. Indeed, it is clear that (22) is not globally exponentially stable since its vector field is bounded in norm by the constant 91.

\subsection{Stability for all $\alpha>0$ : a pendulum example}

Our constructions also apply to the slowly time-varying pendulum dynamics PA2]

$$
\begin{aligned}
& \dot{x}_{1}=x_{2} \\
& \dot{x}_{2}=-x_{1}-\left[1+b_{2}(t / \alpha) m(x, t)\right] x_{2}
\end{aligned}
$$

assuming

(P1) $m: \mathbb{R}^{2} \times \mathbb{R} \rightarrow[0,1]$ is Lipschitz continuous; and

$(\mathcal{P} 2) \quad b_{2}: \mathbb{R} \rightarrow(-\infty, 0]$ is globally bounded, and there are positive constants $T$ and $c_{b}$ such that $5+T \int_{t-T}^{t} b_{2}(l) d l \geq c_{b}$ for all $t \in \mathbb{R}$.

The dynamics (29) was shown to be UGAS for certain choices of the function $b_{2}$ in [PA1] see [K] for related results, and [S1] for results that are restricted to the linear case. However, these earlier results do not lead to explicit Lyapunov functions for (29). In order to build Lyapunov functions for (29) for large constants $\alpha>0$, we use the following observation for the corresponding frozen dynamics $f(x, t, \tau):=\left(x_{2},-x_{1}-[1+\tau m(x, t)] x_{2}\right)$ : 
Lemma 14 The function $V(x):=x_{1}^{2}+x_{2}^{2}+x_{1} x_{2}$ satisfies $\nabla V(x) f(x, t, \tau) \leq-[1+5 \tau] V(x)$ for all $x \in \mathbb{R}^{n}, t \in \mathbb{R}$, and $\tau \leq 0$.

Proof: By grouping terms, one easily shows that

$$
\nabla V(x) f(x, t, \tau)=-V(x)-2 \tau m(x, t) x_{2}^{2}-\tau m(x, t) x_{1} x_{2}
$$

everywhere. Since

$$
V \geq x_{1}^{2}+x_{2}^{2}-\left|x_{1} x_{2}\right| \geq \frac{1}{2} x_{1}^{2}+\frac{1}{2} x_{2}^{2} \geq\left|x_{1} x_{2}\right|
$$

everywhere, we get

$$
-2 \tau m(x, t) x_{2}^{2} \leq-4 \tau m(x, t) V(x), \quad-\tau m(x, t) x_{1} x_{2} \leq-\tau m(x, t) V(x)
$$

everywhere. The lemma follows by substituting (31) into (30) and recalling that $0 \leq m(x, t) \leq 1$ everywhere.

The following is an immediate consequence of Lemma 14, the proof of Theorem 6, and the fact that $V(x):=x_{1}^{2}+x_{2}^{2}+x_{1} x_{2}$ only depends on $x$ :

Theorem 15 Let (29) satisfy $(\mathcal{P} 1)-(\mathcal{P} 2)$. Then (29) has the Lyapunov function

$$
V_{\alpha}^{\sharp}(t, x):=e^{\frac{5 \alpha}{T} \int_{\frac{t}{\alpha}-T}^{\frac{t}{\alpha}} \int_{s}^{\frac{t}{\alpha}} b_{2}(l) d l d s}\left(x_{1}^{2}+x_{2}^{2}+x_{1} x_{2}\right)
$$

for each choice of the constant $\alpha>0$. Hence, (29) is UGAS for all constants $\alpha>0$.

\subsection{Friction example revisited}

We next illustrate Theorem [6 using the one degree-of-freedom mass-spring system [DDNZ, MMD]. The mass-spring system arises in the control of mechanical systems in the presence of friction. However, in contrast to [MMD] where the dynamics are assumed to be rapidly time-varying, here we consider the case where the dynamics are slowly time-varying. While slowly time-varying dynamics can be transformed into rapidly time-varying dynamics by rescaling time, doing so for the slowly time-varying mass spring system produces a new dynamic that does not lend itself to the known methods; see Remark 8 above for details. For this reason, we directly apply the slowly time-varying theory we developed in the preceding sections.

Let us recall the model [MMD]. The dynamics are given by

$$
\begin{aligned}
& \dot{x}_{1}=x_{2} \\
& \dot{x}_{2}=-\sigma_{1}(t / \alpha) x_{2}-k(t) x_{1}-\left\{\sigma_{2}(t / \alpha)+\sigma_{3}(t / \alpha) e^{-\beta_{1} \mu\left(x_{2}\right)}\right\} \operatorname{sat}\left(x_{2}\right)
\end{aligned}
$$

where $x_{1}$ and $x_{2}$ are the mass position and velocity, respectively; $\sigma_{i}:[0, \infty) \rightarrow(0,1], i=1,2,3$ denote positive time-varying viscous, Coulomb, and static friction-related coefficients, respectively; $\beta_{1}$ is a positive constant corresponding to the Stribeck effect; $\mu(\cdot)$ is a positive definite function also related to the Stribeck effect; $k$ denotes a positive time-varying spring stiffness-related coefficient; and sat $(\cdot)$ denotes any continuous function having these properties:

$$
\begin{aligned}
& \text { (a) } \operatorname{sat}(0)=0, \quad \text { (b) } \xi \operatorname{sat}(\xi) \geq 0 \quad \forall \xi \in \mathbb{R}, \\
& \text { (c) } \lim _{\xi \rightarrow+\infty} \operatorname{sat}(\xi)=+1, \quad \text { (d) } \lim _{\xi \rightarrow-\infty} \operatorname{sat}(\xi)=-1
\end{aligned}
$$


Following [MMD], we model the saturation differentiably as

$$
\operatorname{sat}\left(x_{2}\right)=\tanh \left(\beta_{2} x_{2}\right),
$$

where $\beta_{2}$ is a large positive constant, so $\left|\operatorname{sat}\left(x_{2}\right)\right| \leq \beta_{2}\left|x_{2}\right|$ for all $x_{2} \in \mathbb{R}$. However, unlike [MMD], we assume the friction coefficients vary in time slower than the spring stiffness coefficient so we restrict to cases where $\alpha>1$. We are going to establish the stability of (33) and construct corresponding Lyapunov functions $V_{\alpha}$ when the constant $\alpha>0$ is sufficiently large.

Our precise mathematical assumptions on (33) are: $k$ and the $\sigma_{i}$ 's are $C^{1}$ functions with bounded derivatives; $\mu$ has a globally bounded derivative; and there exist constants $c_{b}, T>0$ such that

$$
\int_{t-T}^{t} \sigma_{1}(r) d r \geq c_{b} \quad \forall t \geq 0
$$

We also assume this additional condition whose physical interpretation is that the spring stiffness is nonincreasing:

$$
\exists k_{o}, \bar{k}>0 \text { s.t. } k_{o} \leq k(t) \leq \bar{k} \text { and } k^{\prime}(t) \leq 0 \quad \forall t \geq 0 .
$$

The frozen dynamics $\dot{x}=f(x, t, \tau)$ for (33) are

$$
\begin{aligned}
& \dot{x}_{1}=x_{2} \\
& \dot{x}_{2}=-\tau_{1} x_{2}-k(t) x_{1}-\left\{\tau_{2}+\tau_{3} e^{-\beta_{1} \mu\left(x_{2}\right)}\right\} \operatorname{sat}\left(x_{2}\right)
\end{aligned}
$$

where $\tau=\left(\tau_{1}, \tau_{2}, \tau_{3}\right) \in[0,1]^{3}$ is now a vector of parameters. We apply our construction from Theorem 6 with $p(t)=\left(\sigma_{1}(t), \sigma_{2}(t), \sigma_{3}(t)\right)$ and the function

$$
V(x, t, \tau)=A\left(k(t) x_{1}^{2}+x_{2}^{2}\right)+\tau_{1} x_{1} x_{2} \quad \text { where } A=1+\frac{k_{o}}{2}+\frac{\left(1+2 \beta_{2}\right)^{2}}{k_{o}} .
$$

We first verify the conditions of Assumption 5. Since $A \geq \max \left\{1,1 / k_{o}\right\}$ and $\tau_{1} \leq 1$, we have

$$
\frac{1}{2}\left(x_{1}^{2}+x_{2}^{2}\right) \leq V(x, t, \tau) \leq A^{2} \bar{k}\left(\left|x_{1}\right|+\left|x_{2}\right|\right)^{2} \leq 2 A^{2} \bar{k}|x|^{2}
$$

everywhere. Let us now compute $\dot{V}(x, t, \tau)$ for all values $\tau \in[0,1]^{3}$. Since $k^{\prime}(t) \leq 0$ everywhere, this gives

$$
\begin{aligned}
\dot{V}(x, t, \tau) & \leq V_{x}(x, t, \tau) f(x, t, \tau) \\
& =\left[2 A k(t) x_{1}+\tau_{1} x_{2}\right] x_{2}-\left[2 A x_{2}+\tau_{1} x_{1}\right]\left\{\tau_{1} x_{2}+\left[\tau_{2}+\tau_{3} e^{-\beta_{1} \mu\left(x_{2}\right)}\right] \operatorname{sat}\left(x_{2}\right)+k(t) x_{1}\right\} .
\end{aligned}
$$

Therefore, by grouping and canceling terms, we also have

$$
\begin{aligned}
\dot{V}(x, t, \tau) & \leq-\tau_{1} k_{0} x_{1}^{2}-\left(2 A \tau_{1}-\tau_{1}\right) x_{2}^{2}+\tau_{1}\left(1+2 \beta_{2}\right)\left|x_{1} x_{2}\right| \\
& \leq-\tau_{1} \frac{k_{o}}{2}|x|^{2}-\left[\tau_{1} \frac{k_{o}}{2} x_{1}^{2}+(A-1 / 2) \tau_{1} x_{2}^{2}-\tau_{1}\left(1+2 \beta_{2}\right)\left|x_{1} x_{2}\right|\right] \\
& =-\tau_{1} \frac{k_{o}}{2}|x|^{2}-\tau_{1} \frac{k_{o}}{2}\left(\left|x_{1}\right|-\frac{1+2 \beta_{2}}{k_{o}}\left|x_{2}\right|\right)^{2}+\left(\frac{\tau_{1}\left(1+2 \beta_{2}\right)^{2}}{2 k_{o}}+\frac{\tau_{1}}{2}-A \tau_{1}\right) x_{2}^{2} \\
& \leq-\frac{\tau_{1} k_{o}}{4 A^{2} \bar{k}} V(x, t, \tau)
\end{aligned}
$$

where the first inequality follows from (34) (b), the inequality $\left|\operatorname{sat}\left(x_{2}\right)\right| \leq \beta_{2}\left|x_{2}\right|$, and the fact that $\tau_{i} \in[0,1]$ for each $i$; the second inequality uses the fact that $A-1 / 2 \geq k_{o} / 2$; and the last inequality 
is from the choice of $A$ and the bounds (39). Hence, Assumption 5 of Theorem 6 readily follows from (36) with the choices

$$
q(\tau)=\frac{\tau_{1} k_{o}}{4 A^{2} \bar{k}}, \quad c_{a}=1
$$

We conclude as follows:

Corollary 16 Under the preceding assumptions, there exists a constant $\alpha_{o}>0$ such that for all constants $\alpha>\alpha_{o}$, the system (33) is UGAS and admits the Lyapunov function

$$
V_{\alpha}(t, x):=V(x, t, p(t / \alpha)) e^{\frac{\alpha \bar{b}}{T} \int_{\frac{t}{\alpha}-T}^{\frac{t}{\alpha}} \int_{s}^{\frac{t}{\alpha}} \sigma_{1}(l) d l d s}
$$

where $V$ is the function defined in (38), $\bar{b}=k_{o} /\left(4 A^{2} \bar{k}\right)$, and $p(t)=\left(\sigma_{1}(t), \sigma_{2}(t), \sigma_{3}(t)\right)$.

\subsection{Identification dynamics revisited}

Our Lyapunov function constructions also apply to the slowly time-varying dynamics

$$
\dot{x}=h(t / \alpha) m(t) m^{\top}(t) x, \quad x \in \mathbb{R}^{n}
$$

assuming there are positive constants $T, \tilde{c}, \underline{\alpha}$, and $\bar{\alpha}$ such that

$(\mathcal{I} 1) h: \mathbb{R} \rightarrow[-\bar{\alpha}, 0]$ is continuous with a bounded first derivative and $\int_{t-T}^{t} h(r) d r \leq-\underline{\alpha}$ for all $t \in \mathbb{R}$.

(I2) $m: \mathbb{R} \rightarrow \mathbb{R}^{n}$ is continuous and satisfies $|m(t)| \equiv 1$ and $\underline{\alpha} I \leq \int_{t}^{t+\tilde{c}} m(r) m^{\top}(r) d r \leq \bar{\alpha} I$ for all $t \in \mathbb{R}$.

where $I$ is the identity matrix and for matrices $A, B \in \mathbb{R}^{n \times n}$ we use $B-A \geq 0$ to mean that $B-A$ is positive semi-definite. See also Remark 23 below for the generalization of our result to control affine systems

$$
\dot{x}=h(t / \alpha) m(t) m^{\top}(t) x+g(x, t, t / \alpha) u
$$

for suitable matrix valued functions $g$. The particular case $\dot{x}=-m(t) m^{\top}(t) x$ of (41) has been studied extensively in the context of identification theory [MMD, PA1]. In [MMD], we showed how to construct explicit Lyapunov functions for the rapidly time-varying system $\dot{x}=f(\alpha t) m(t) m^{\top}(t) x$ for appropriate nonpositive functions $f$ and large positive constants $\alpha$. However, these earlier results do not lead to explicit Lyapunov functions for the slowly time-varying dynamics (41) for large constants $\alpha>0$; see Remark 8 above. Instead, we construct Lyapunov functions for (41) using the following analogue of [MMD, Lemma 6]:

Lemma 17 Assume there are positive constants $T, \tilde{c}, \underline{\alpha}$, and $\bar{\alpha}$ such that $(\mathcal{I} 1)-(\mathcal{I} 2)$ are satisfied and set

$$
P(t, \tau)=\kappa I-\tau \int_{t-\tilde{c}}^{t} \int_{s}^{t} m(l) m^{T}(l) \mathrm{d} l \mathrm{~d} s, \text { where } \kappa=\frac{\tilde{c}}{2}+\frac{\bar{\alpha}^{2} \tilde{c}^{4}}{2 \underline{\alpha}}+\tilde{c}^{2} .
$$

Then for each $\tau \in[-\bar{\alpha}, 0]$ the function

$$
V(x, t, \tau)=x^{\top} P(t, \tau) x
$$

satisfies the requirements of Assumption 5 for the frozen dynamics $f(x, t, \tau)=\tau m(t) m^{\top}(t) x$ and $p(s)=h(s)$. 
Proof: We first apply Lemma $4(\mathrm{~B})$ to $\Theta(t)=x^{\top} m(t) m^{\top}(t) x$ for each $x$ and $\tau \in[-\bar{\alpha}, 0]$ to get

$$
\frac{\partial V}{\partial t}(x, t, \tau)=-\tau \tilde{c} x^{\top} m(t) m^{\top}(t) x+\tau x^{\top}\left[\int_{t-\tilde{c}}^{t} m(l) m^{\top}(l) d l\right] x
$$

and

$$
\frac{\partial V}{\partial x}(x, t, \tau) f(x, t, \tau)=2 \tau x^{\top}\left[\kappa I-\tau \int_{t-\tilde{c}}^{t} \int_{s}^{t} m(l) m^{T}(l) \mathrm{d} l \mathrm{~d} s\right] m(t) m^{\top}(t) x
$$

so the derivative $\dot{V}=\frac{\partial V}{\partial t}+\frac{\partial V}{\partial t} f$ along the trajectories of $f$ satisfies

$$
\begin{aligned}
\dot{V} & \leq \tau\left[(2 \kappa-\tilde{c})\left|m^{\top}(t) x\right|^{2}+\underline{\alpha}|x|^{2}\right]+\tau^{2}|x|\left|m^{\top}(t) x\right| \tilde{c}^{2} \\
& =\tau\left\{(2 \kappa-\tilde{c})\left|m^{\top}(t) x\right|^{2}+\underline{\alpha}|x|^{2}+\tau|x|\left|m^{\top}(t) x\right| \tilde{c}^{2}\right\}
\end{aligned}
$$

where the inequality follows from Lemma $4(\mathrm{~A}),(\mathcal{I} 2)$, and the facts that $|m(t)| \equiv 1$ and $\tau \leq 0$. Set

$$
\omega=\frac{\underline{\alpha}}{2 \tilde{c}^{2} \bar{\alpha}} .
$$

Then the triangle inequality gives

$$
\left|m^{\top}(t) x\right||x| \leq \omega|x|^{2}+\frac{1}{4 \omega}\left|m^{\top}(t) x\right|^{2}
$$

so since $\tau \leq 0$, we get

$$
\begin{aligned}
& (2 \kappa-\tilde{c})\left|m^{\top}(t) x\right|^{2}+\underline{\alpha}|x|^{2}+\tau|x|\left|m^{\top}(t) x\right| \tilde{c}^{2} \\
& \geq(2 \kappa-\tilde{c})\left|m^{\top}(t) x\right|^{2}+\underline{\alpha}|x|^{2}+\omega \tau \tilde{c}^{2}|x|^{2}+\frac{\tau \tilde{c}^{2}}{4 \omega}\left|m^{\top}(t) x\right|^{2} \\
& =\left[2 \kappa-\tilde{c}+\frac{\tau \tilde{c}^{2}}{4 \omega}\right]\left|m^{\top}(t) x\right|^{2}+\left(\underline{\alpha}+\omega \tau \tilde{c}^{2}\right)|x|^{2} \\
& \geq \frac{\underline{\alpha}|x|^{2}}{2}
\end{aligned}
$$

by our choices of $\kappa$ and $\omega$. This and (43) give $\dot{V} \leq \frac{\tau \underline{\alpha}}{2}|x|^{2}$ everywhere; and Lemma 4 (A), the fact that $|m(t)| \equiv 1$, and our choice of $\kappa$ give

$$
\begin{aligned}
& \kappa|x|^{2} \leq V(x, t, \tau) \leq\left(\kappa+\tilde{c}^{2} \bar{\alpha}\right)|x|^{2} \\
& \left|\frac{\partial V}{\partial \tau}(x, t, \tau)\right| \leq \frac{\tilde{c}^{2}}{2}|x|^{2} \leq V(x, t, \tau) .
\end{aligned}
$$

Hence,

$$
\dot{V}(x, t, \tau) \leq-q(\tau) V(x, t, \tau), \text { where } q(\tau):=-\frac{\tau \underline{\alpha}}{2\left(\kappa+\tilde{c}^{2} \bar{\alpha}\right)}
$$

everywhere. Therefore, we can satisfy the requirements of Assumption 5 using $p(s)=h(s)$ and

$$
\alpha_{1}(s)=\kappa s^{2}, \quad \alpha_{2}(s)=\left(\kappa+\tilde{c}^{2} \bar{\alpha}\right) s^{2}, \quad c_{a}=1, \quad c_{b}=\frac{\underline{\alpha}^{2}}{2\left(\kappa+\tilde{c}^{2} \bar{\alpha}\right)} .
$$

This proves the lemma.

The following is an immediate consequence of the preceding lemma and Theorem 6 . 
Theorem 18 Let (41) admit positive constants $T, \tilde{c}, \underline{\alpha}$, and $\bar{\alpha}$ such that $(\mathcal{I} 1)-(\mathcal{I} 2)$ are satisfied and choose $c_{b}$ as in (44). Then for any constant $\alpha \geq 2 T \sup \left\{\left|h^{\prime}(r)\right|: r \in \mathbb{R}\right\} / c_{b}$, the function

$$
V_{\alpha}^{\sharp}(t, x):=e^{-\frac{\alpha \underline{\alpha}}{2 T\left(\kappa+\tilde{c}^{2} \bar{\alpha}\right)}} \int_{\frac{t}{\alpha}-T}^{\frac{t}{\alpha}} \int_{s}^{\frac{t}{\alpha}} h(l) d l d s
$$

with $V(x, t, \tau)=x^{\top} P(t, \tau) x$ as defined in Lemma 17 is a Lyapunov function for (41). Hence, (41) is $U G A S$ for all constants $\alpha \geq 2 T \sup \left\{\left|h^{\prime}(r)\right|: r \in \mathbb{R}\right\} / c_{b}$.

\section{Input-to-State Stability}

We next extend our results to control affine slowly time-varying systems

$$
\dot{x}=f(x, t, p(t / \alpha))+g(x, t, p(t / \alpha)) u
$$

evolving on $\mathbb{R}^{n}$ with control values $u \in \mathbb{R}^{m}$, where $f: \mathbb{R}^{n} \times[0, \infty) \times \mathbb{R}^{d} \rightarrow \mathbb{R}^{n}$ and $g: \mathbb{R}^{n} \times[0, \infty) \times$ $\mathbb{R}^{d} \rightarrow \mathbb{R}^{n \times m}$ are locally Lipschitz functions that admit $\alpha_{4} \in \mathcal{K}_{\infty}$ such that

$$
|f(x, t, p(t / \alpha))|+|g(x, t, p(t / \alpha))| \leq \alpha_{4}(|x|)
$$

everywhere, and where $p: \mathbb{R} \rightarrow \mathbb{R}^{d}$ for some $d$ is bounded with a bounded first derivative. The control functions (i.e. inputs) for (46) comprise the set $\mathcal{U}$ of all measurable essentially bounded functions $\mathbf{u}:[0, \infty) \rightarrow \mathbb{R}^{m}$ with the essential supremum norm $|\cdot|_{\infty}$. We assume throughout this section that Assumption 5 holds for some function $V \in C^{1}$ and that $\alpha_{1} \in \mathcal{K}_{\infty}$ and $c_{a}$ from Assumption 5 are also such that

$$
\begin{aligned}
& A_{5}\left|V_{x}(x, t, p(t / \alpha))\right| \leq c_{a} \sqrt{\alpha_{1}(|x|)} \\
& A_{6}|g(x, t, p(t / \alpha))| \leq c_{a}\left(1+\sqrt[4]{\alpha_{1}(|x|)}\right)
\end{aligned}
$$

hold for all $t \geq 0, \alpha>0$, and $x \in \mathbb{R}^{n}$. Notice that $A_{5}$ reduces to a linear growth condition when $\alpha_{1}(x)=|x|^{2}$ and so automatically holds in the classical case where $V$ has the form $x^{\top} P(t) x$ for a suitable bounded positive definite matrix. We show that when Assumption 5 and $A_{5}-A_{6}$ hold, and when the constant $\alpha>0$ is sufficiently large, the control system (46) satisfies the input-to-state stable (ISS) property and admits the ISS Lyapunov function (5). We first recall the relevant ISS definitions from [ELW, MM2, S2, S3].

For a general locally Lipschitz control affine system

$$
\dot{x}=h(x, t)+J(x, t) u
$$

where $h$ and $J$ are uniformly state bounded (as defined in Section 21), and for given values $t_{o} \geq 0$, $x_{o} \in \mathbb{R}^{n}$, and $\mathbf{u} \in \mathcal{U}$, we let $t \mapsto \phi\left(t ; t_{o}, x_{o}, \mathbf{u}\right)$ denote the unique maximal solution of the initial value problem

$$
\dot{x}(t)=h(x(t), t)+J(x(t), t) \mathbf{u}(t) \text { a.e. } t, \quad x\left(t_{o}\right)=x_{0} .
$$

We always assume all trajectories $\phi\left(\cdot ; t_{o}, x_{o}, \mathbf{u}\right)$ so defined are defined on all of $\left[t_{o}, \infty\right)$. Later we specialize to the controlled system (46) for fixed constants $\alpha>0$.

Definition 19 We say that (47) is input-to-state stable (ISS) provided there exist $\beta \in \mathcal{K} \mathcal{L}$ and $\gamma \in \mathcal{K}_{\infty}$ such that

$$
\left|\phi\left(t ; t_{o}, x_{o}, \mathbf{u}\right)\right| \leq \beta\left(\left|x_{o}\right|, t-t_{o}\right)+\gamma\left(|\mathbf{u}|_{\infty}\right)
$$

holds for all $t \geq t_{o}, t_{o} \geq 0, x_{o} \in \mathbb{R}^{n}$, and $\mathbf{u} \in \mathcal{U}$. 
Definition 20 A smooth function $W: \mathbb{R}^{n} \times[0, \infty) \rightarrow[0, \infty)$ is called an ISS Lyapunov function for (47) provided there exist functions $\alpha_{1}, \alpha_{2}, \chi \in \mathcal{K}_{\infty}$ and a positive definite function $\alpha_{3}$ such that

(1) $\alpha_{1}(|x|) \leq W(x, t) \leq \alpha_{2}(|x|)$ and

(2) $|u| \leq \chi(|x|)$ implies $W_{t}(x, t)+W_{x}(x, t)[h(x, t)+J(x, t) u] \leq-\alpha_{3}(|x|)$.

hold for all $x \in \mathbb{R}^{n}, t \geq 0$, and $u \in \mathbb{R}^{m}$.

The following lemma comes from [ELW]:

Lemma 21 If (47) has an ISS Lyapunov function, then it is ISS.

We prove the following analogue of [MMD, Theorem 4]:

Theorem 22 Assume (4) satisfies Assumption 5. Assume $A_{5}-A_{6}$ everywhere hold where $c_{a}, V$, and $\alpha_{1}$ are chosen as in Assumption 5. Then for each constant $\alpha>4 T c_{a} \bar{p} / c_{b}$, the dynamics (46) are ISS and

$$
V_{\alpha}^{\sharp}(t, x):=e^{\frac{\alpha}{T} \int_{\frac{t}{\alpha}-T}^{\frac{t}{\alpha}} \int_{s}^{\frac{t}{\alpha}} q(p(l)) d l d s} V(x, t, p(t / \alpha))
$$

is an ISS Lyapunov function for (46).

Proof: We indicate the changes needed in the proof of Theorem 6. Consider the function $\chi \in \mathcal{K}_{\infty}$ defined by

$$
\chi(s):=\frac{c_{b} \sqrt{\alpha_{1}(s)}}{2 T c_{a}^{2}\left(1+\sqrt[4]{\alpha_{1}(s)}\right)},
$$

where $\alpha_{1}$ and $c_{a}$ are as in Assumption 5. This function is of class $\mathcal{K}_{\infty}$ since $s \mapsto \alpha_{1}^{1 / 4}(s)$ and $s \mapsto s^{2} /(1+s)$ are both $\mathcal{K}_{\infty}$. Our assumptions imply that if $|u| \leq \chi(|x|)$, then

$$
\begin{aligned}
\mid V_{x}(x, t, p(t / \alpha)) g(x, t, p(t / \alpha) u \mid & \leq \frac{c_{b} \alpha_{1}(|x|)}{2 T} \\
& \leq \frac{c_{b}}{2 T} V(x, t, p(t / \alpha))
\end{aligned}
$$

everywhere. Define $\hat{V}$ as in (7) and $E(t, \alpha)$ as in (8). Then, along any trajectory $x=\phi(t)$ of (46) with inputs $\mathbf{u}$ satisfying $|\mathbf{u}|_{\infty} \leq \chi(|\phi(t)|)$ everywhere, we get

$$
\dot{\hat{V}}=-q(p(t / \alpha)) \hat{V}(t, x)+\left[\frac{c_{a} \bar{p}}{\alpha}+\frac{c_{b}}{2 T}\right] \hat{V}(x, t)
$$

everywhere and therefore (by reasoning exactly as in Section 4) also

$$
\begin{aligned}
\dot{V}_{\alpha}^{\sharp} & \leq E(t, \alpha)\left[\frac{c_{a} \bar{p}}{\alpha}+\frac{c_{b}}{2 T}-\frac{1}{T} \int_{t / \alpha-T}^{t / \alpha} q(p(l)) d l\right] \hat{V}(x, t) \\
& \leq E(t, \alpha)\left[\frac{c_{a} \bar{p}}{\alpha}-\frac{c_{b}}{2 T}\right] \hat{V}(x, t) \leq-\frac{c_{b} E(t, \alpha)}{4 T} \hat{V}(x, t)
\end{aligned}
$$

when $\alpha>4 T c_{a} \bar{p} / c_{b}$, where the second inequality follows from $A_{4}$, and the last inequality follows from our choice of $\alpha$. We then argue exactly as before to show that $V_{\alpha}^{\sharp}$ is an ISS Lyapunov function for (46) for all constants $\alpha>4 T c_{a} \bar{p} / c_{b}$. The theorem now follows from Lemma 21, 
Remark 23 Theorem 22 readily applies to our friction example from Section 6.3 above, showing that (40) is actually an ISS Lyapunov function for the slowly time-varying controlled friction dynamic

$$
\begin{aligned}
& \dot{x}_{1}=x_{2} \\
& \dot{x}_{2}=-\sigma_{1}(t / \alpha) x_{2}-k(t) x_{1}+g(x, t, t / \alpha) u-\left\{\sigma_{2}(t / \alpha)+\sigma_{3}(t / \alpha) e^{-\beta_{1} \mu\left(x_{2}\right)}\right\} \operatorname{sat}\left(x_{2}\right)
\end{aligned}
$$

for any $g$ satisfying our assumption $A_{6}$ for some $c_{a}>0$ and with $\alpha_{1}(s)=s^{2} / 2$, provided the constants $c_{a}>0$ and $\alpha>0$ are sufficiently large. Similar extensions can be made for the other examples we considered above.

\section{Conclusion}

We provided general conditions under which slowly time-varying systems are uniformly globally asymptotically stable and input-to-state stable with respect to general perturbations, thus extending [PA2, S1] to situations where the corresponding frozen dynamics are not necessarily exponentially stable. Moreover, we provided new methods for constructing explicit closed form strict ISS Lyapunov functions for slowly time-varying control systems in terms of a suitable family of generalized Lyapunov like functions for the frozen dynamics. This is significant because Lyapunov functions play essential roles in robustness analysis and controller design.

We conjecture that our work can be extended to systems that are subjected to actuator or measurement errors, or which are components of larger controlled hybrid dynamical systems. It would also be of interest to extend our work to slowly time-varying systems with outputs and to construct corresponding input-to-output stable (IOS) Lyapunov functions; see [SW1, SW2] for further background on systems with outputs and [MM1] for some first results on constructing IOS Lyapunov functions for non-autonomous systems in terms of given nonstrict Lyapunov functions. We leave these extensions for future papers.

\section{Acknowledgements}

This research was supported by NSF Grant 0424011. This work was done while the first author visited Louisiana State University (LSU). He thanks LSU for the kind hospitality he enjoyed during this period. Both authors thank Marcio de Queiroz and Patrick De Leenheer for illuminating discussions.

\section{References}

[A] D. Angeli, Input-to-State Stability of PD-controlled robotic systems, Automatica 35 (1999), 1285-1290.

[AS] D. Angeli and E.D. Sontag, Forward completeness, unboundedness observability, and their Lyapunov characterizations, Systems and Control Letters 38 (1999), 209-217.

[ASW] D. Angeli, E.D. Sontag, and Y. Wang, A characterization of integral input to state stability, IEEE Trans. Automatic Control 45 (2000), 1082-1097.

[DDNZ] M.S. de Queiroz, D.M. Dawson, S. Nagarkatti, and F. Zhang, Lyapunov-Based Control of Mechanical Systems, Birkhäuser, Cambridge, MA, 2000. 
[ELW] H. Edwards, Y. Lin, and Y. Wang, On input-to-state stability for time-varying nonlinear systems, in Proceedings of the 39th IEEE Conf. on Decision and Control, Sydney, Australia, December 2000, 3501-3506.

[K] H. Khalil, Nonlinear Systems, Third Edition, Prentice Hall, Englewood Cliffs, NJ, 2002.

[MM1] M. Malisoff and F. Mazenc, Further constructions of strict Lyapunov functions for timevarying systems, in Proceedings of the American Control Conference, Portland, OR, June, 2005, 1889-1894.

[MM2] M. Malisoff and F. Mazenc, Further remarks on strict input-to-state stable Lyapunov functions for time-varying systems, Automatica 41 (2005), 1973-1978.

[M] F. Mazenc, Strict Lyapunov functions for time-varying systems, Automatica 39 (2003), 349-353.

[MM3] F. Mazenc and M. Malisoff, Further constructions of control-Lyapunov functions and stabilizing feedbacks for systems satisfying the Jurdjevic-Quinn conditions, IEEE Trans. Automatic Control 51 (2006), 360-365.

[MMD] F. Mazenc, M. Malisoff, and M. de Queiroz, Further results on strict Lyapunov functions for rapidly time-varying nonlinear systems, Automatica 42 (2006), 1663-1671.

[PA1] J. Peuteman and D. Aeyels, Exponential stability of nonlinear time-varying differential equations and partial averaging, Mathematics of Control, Signals, and Systems 15 (2002), 42-70.

[PA2] J. Peuteman and D. Aeyels, Exponential stability of slowly time-varying nonlinear systems, Mathematics of Control, Signals, and Systems 15 (2002), 202-228.

[S1] V. Solo, On the stability of slowly time-varying linear systems, Mathematics of Control, Signals, and Systems 7 (1994), 331-350.

[S2] E.D. Sontag, Smooth stabilization implies coprime factorization, IEEE Trans. Automatic Control 34 (1989), 435-443.

[S3] E.D. Sontag, The ISS philosophy as a unifying framework for stability-like behavior, in Nonlinear Control in the Year 2000, Volume 2, A. Isidori, F. Lamnabhi-Lagarrigue, and W. Respondek (eds.), Springer-Verlag, Berlin, 2000, 443-468.

[SW1] E.D. Sontag and Y. Wang, Notions of input to output stability, Systems and Control Letters 38 (1999), 235-248.

[SW2] E.D. Sontag and Y. Wang, Lyapunov characterizations of input to output stability, SIAM J. Control and Optimization 39 (2001), 226-249. 